\title{
ENSAYO DE TALA CON UN HACHA DE PIEDRA PULIDA
}

\author{
POR
}

\author{
RAMON FABREGAS VALCARCE (*)
}

\begin{abstract}
RESUMEN Se lleva a cabo un experimento de corte de árboles con un hacha fabricada "ex profeson, de características similares a ejemplares recuperados en contextos arqueológicos de Galicia. A pesar de que el enmangue se reveló poco seguro, el hacha efectuó el trabajo con notable eficacia y sin acusar un desgaste significativo.
\end{abstract}

\begin{abstract}
Tree-felling with a ground-stone axe. An experiment of tree-felling was carried out with a ground-stone axe made for this purpose, resembling the morphological features of some Galician archaeological examples. While the hafting posed problems, the axe blade worked very efficiently indeed, without showing any significant wear afterwards.
\end{abstract}

Palabras clave Experimentos de tala. Análisis funcional. Desgaste. Eficacia de hachas de piedra.

Key words Tree-Felling. Functional analysis. Wear. Stone axe efficiency

La introducción y generalización de la economía de producción en muchas regiones de Europa va acompañada de una significativa reducción de las áreas boscosas, con el fin de ampliar las superficies aptas para la puesta en cultivo o facilitar la expansión de plantas para alimentar a los animales. En este proceso de deforestación juega un papel fundamental el fuego, pero también las hachas pulimentadas, empleadas en la tala de los árboles o en el anillado de éstos (remoción de la capa de cambium en la periferia del tronco, provocando así la muerte de la planta). Por todo ello no es de extrañar que en los registros arqueológicos de yacimientos neolíticos adquiera un papel relevante esta clase de útiles pulimentados, así como tipos morfotipológicamente próximos (v.g. azuelas). La región Noroeste de la Península no es una excepción a esta pauta y en los ajuares tumulares (casi la única representación conocida en la actualidad de las poblaciones neolíticas locales) son las hachas el elemento más universalmente distribuido, así como de los más significativos desde el punto de vista cuantitativo (Fábregas, 1991).

Teniendo en cuenta lo anterior, no es de extrañar que, ya en los comienzos mismos de la ciencia prehistórica, algunos autores se interrogasen sobre la función o la eficiencia de un utillaje pulimen-

(`) Departamento de Historia 1, Area de Prehistoria. Universidad de Santiago de Compostela. 15703. La Coruña. 
tado tan llamativo y frecuente en los registros arqueológicos desde el Neolítico. La respuesta a estas cuestiones vino dada mediante la comparación con herramientas actuales empleadas tanto por poblaciones primitivas como en nuestra propia cultura, así como a través de la experimentación con réplicas o piezas originales. En este último método se destacan desde un principio los arqueólogos anglosajones y de la Europa septentrional, arrancando desde las tempranas observaciones efectuadas por Nilsson (1868) en los años 40 del siglo pasado, pasando por las experiencias de Sehested (1884) o Quente (1914) hasta llegar a Sonnenfeld (1962) u Olausson (1982, 1982-83). Son especialmente interesantes los experimentos de tala efectuados en el bosque de Draved durante la década de los cincuenta, dentro de un proyecto de dimensiones más amplias destinado a investigar las antiguas técnicas agrícolas (Iversen, 1956; Jørgensen, 1985). A este respecto, hay que citar también los trabajos experimentales llevados a cabo por un equipo de la Universidad de Durham (Harding y Young, 1979).

El estudio complementario del desgaste sobre las hachas experimentales y la eventual identificación de las huellas de uso producidas ha sido comparativamente escaso (Olausson, Sonnenfeld), y los autores se limitaron comúnmente a la detección de estrías u otros estigmas de utilización, interpretándolos funcionalmente a la luz de paralelos etnográficos o de la "lógica». Semenov (1981: 237) recurre en buena medida a la comparación con herramientas metálicas actuales cuando aborda la cuestión del empleo de los diversos útiles pulimentados. El método de Semenov, pionero en todos los órdenes del análisis funcional, ha sido seguido por un gran número de investigadores (Detev, 1960; Kantman 1969-70; Delibes, 1974; Roodenberg, 1982), si bien otros (Cauvin, 1968: 24, Stelc y Malina, 1970: 52) han señalado la dificultad de distinguir las modificaciones superficiales producidas por el proceso de pulimento o reafilado de aquéllas originadas por la utilización, en tanto que algunos investigadores han puesto de relieve la carga de subjetividad que con frecuencia impregna las descripciones del microdesgaste sobre el utillaje de piedra pulida (Madsen, 1984: 56).

En este primer experimento de tala que hemos llevado a cabo se ha utilizado un hacha fabricada expresamente, cuyas características morfotipológicas la asemejan a un tipo común en contextos tumulares o domésticos del Noroeste peninsular. Este trabajo pretendía verificar la eficacia de esta clase de utensilios, así como observar la aparición de desgaste, cuantificando éste. En otro lugar (Fábregas, 1991), hemos expuesto en detalle los resultados del análisis microscópico de la pieza con posterioridad a su empleo, por lo que aquí nos limitaremos a mencionar genéricamente las alteraciones observadas mediante un microscopio binocular de luz incidente (usando entre 50x y 200x).

La pieza utilizada está hecha de una roca (greenstone), formada a partir de un proceso de metamorfismo regional. Sus características morfológicas son las siguientes:

Longitud: $118 \mathrm{~mm}$. Anchura: $64 \mathrm{~mm}$. Espesor: $24 \mathrm{~mm}$. Peso: 265 grs. Forma general: trapezoidal. Caras: curvas convexas. Lados: rectos. Aristas: convergentes y curvilineas. Filo: convexo simétrico. Zona activa: convexa simétrica. Talón: redondeado y apuntado. Sección transversal: biconvexa. Acabado: pulimentado integral total (Fig. 1).

En el proceso de elaboración del hacha se necesitó aproximadamente media hora para lograr la forma general del artefacto mediante percusión. A continuación se procedió al picoteado y pulimento de éste, utilizando como agentes abrasivos arena y agua, tareas que requirieron un total de seis horas. Estas cifras no difieren significativamente de las documentadas en otros experimentos sobre materiales similares (Olausson, 1982-83: 28; Harding, 1983: 41). El mango del hacha estaba hecho en madera de fresno y tenía una longitud total de $609 \mathrm{~mm}$. por una anchura máxima de $58 \mathrm{~mm}$. y un grosor también máximo de $44 \mathrm{~mm}$. Estas dos últimas magnitudes fueron tomadas en el punto donde se había practicado un orificio, de $61 \mathrm{~mm}$, de profundidad, destinado a encajar el hacha; el peso del mango era de 464 grs. Fue realizado en un tiempo de cuatro horas recurriendo para ello a herramientas metálicas, y tanto la madera empleada como el diseño general se corresponden con modelos arqueológicos recuperados en el Centro y Norte de Europa (Müller-Beck, 1963: figs. 1-5; 
Jørgensen, 1983: fig. 11). El hacha fue introducida en el alvéolo (Lám. I, 1) y fijada dentro de éste mediante una combinación de resina de pino y cera de abejas (1).

El árbol escogido para la tala fue el aliso europeo (Alnus glutinosa), especie de hoja perenne presente en toda la Europa atlántica, en tierras húmedas, con frecuencia en las márgenes de los ríos, de porte rectilíneo, alcanzando unos $10 \mathrm{~m}$. de altura por término medio. Su madera es de dureza mediana y resistente a la humedad, siendo preferida en Galicia para la fabricación de los tradicionales zuecos, pero también usada en la elaboración de muebles, para cerrar las fincas o simplemente como combustible. Hasta el momento no tenemos pruebas de la utilización de esta especie arbórea en época prehistórica, entre otras razones por la habitualmente mala conservación del material orgánico debido a las condiciones medioambientales, pero su presencia es muy frecuente en los registros polínicos de gran número de yacimientos arqueológicos gallegos (Aira et alii, 1989).

El experimento tuvo lugar en una finca particular del país de Gales durante un día del mes de Junio de 1985 , con una temperatura atmosférica en torno a los $18^{\circ}$ y gran humedad. El corte de los árboles fue practicado a una altura de aproximadamente $1 \mathrm{~m}$. sobre el nivel del suelo y la técnica utilizada fue la recomendada por diversos especialistas (Müller-Beck, 1965: 179; Jørgensen, 1985: 30), es decir un golpe administrado no con toda la fuerza del hombro, intentando penetrar profundamente, como se realizaría con un hacha de acero, sino actuando fundamentalmente con la articula-
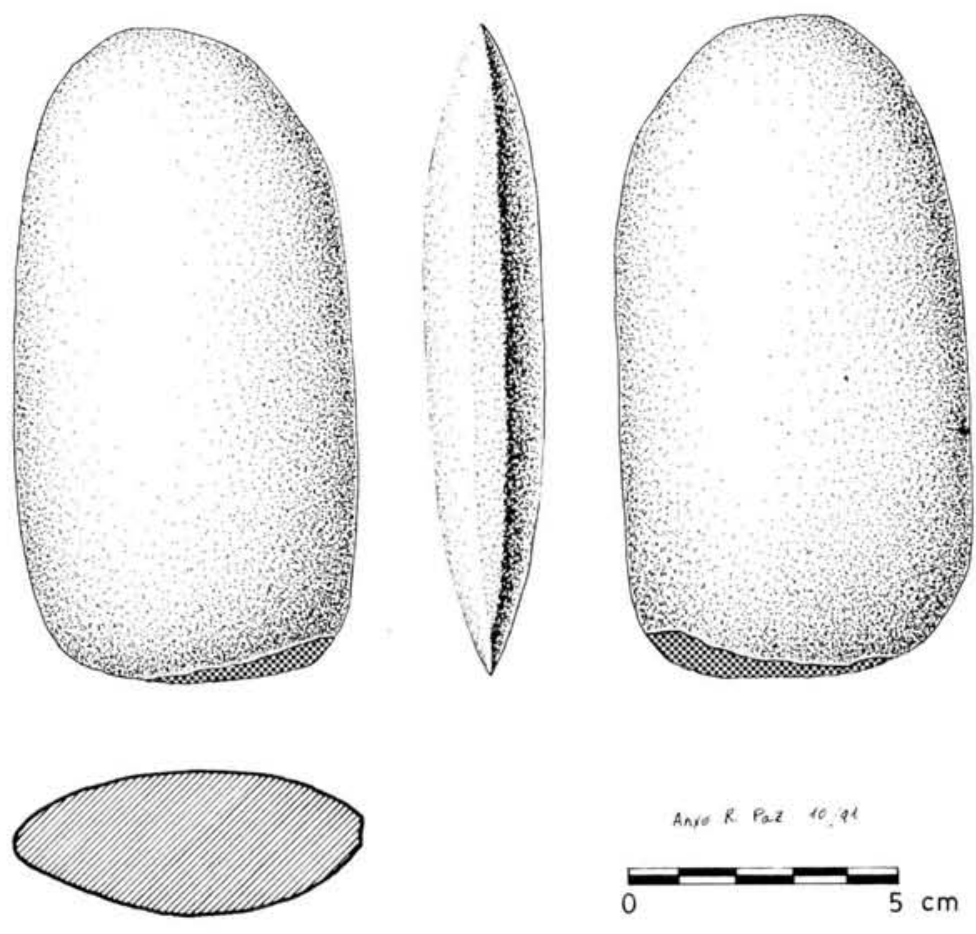

FIG. 1.-Hacha empleada en el experimento. El sombreado señala el ârea de la zona activa que presentaba una pátina lustrosa tras efectuar aquél.

(1) Quiero agradecer a John Lord, del Museo de Grime's Graves (Norfolk, Inglaterra) y a Mark Newcomer (Institute of Archaeology, Londres) la elaboración del hacha y su mango, respectivamente. Un reconocimiento especial debo a Roger Grace por haberme permitido llevar a cabo el trabajo de tala en la finca de su propiedad. 
ción del codo (Lám. I, 2); por otra parte la hoja debe golpear el tronco en un ángulo lo más agudo posible, extrayendo virutas alargadas transversalmente (Lám. II, 1). Tan sólo se cronometró el tiempo de trabajo efectivo y no el empleado en descansar.

En la primera parte del experimento se procedió a la corta de un tronco de $12 \mathrm{~cm}$. de diámetro en 61 minutos. El árbol cayó cuando el tajo había penetrado $8 \mathrm{~cm}$. en línea recta y se habían practicado algunos cortes complementarios a los lados (en esta última tarea se invirtieron 5 minutos). No se observaba desgaste en el filo del hacha a simple vista.

A continuación, se efectuó la corta de un tronco de $18 \mathrm{~cm}$. de diámetro en 60 minutos. El árbol cayó cuando el hacha había penetrado a una profundidad de sólo $7 \mathrm{~cm}$, y tras haberse practicado cortes laterales durante cinco minutos (Lám. II, 2). La razón de esta mayor rapidez en relación con el experimento anterior estriba en que el tronco presentaba una inclinación natural que ayudaba a derribarlo una vez que el corte había alcanzado esas dimensiones, simplemente con la fuerza conjunta de dos hombres. El hacha todavía presentaba el filo en perfectas condiciones.

Por último, se inició el corte de un tronco de $15 \mathrm{~cm}$. de diámetro. A los 13 minutos del comienzo el hacha se salió del alvéolo, observándose una grieta en este último. Se intentó reparar el útil fijándolo mediante una cuña de madera con lo que pudo continuarse la labor durante otros 3 - minutos más. Cuando se abandonó el experimento, el corte había progresado $6 \mathrm{~cm}$. en el interior del árbol (Lám. III, 1). El hacha seguía sin mostrar un desgaste aparente aunque si se observaba un tenue lustre en las áreas inmediatas al filo (Fig. 1 y Lám. III, 2).

En el momento de evaluar la eficacia del hacha de piedra pulida debemos tomar en consideración varios factores que influyen en el resultado final del experimento: para empezar lo limitado de éste debido a los problemas con el enmangue; en segundo lugar la inexperiencia del "leñador» que explica lo dilatado de los tiempos requeridos para acometer la tarea, tiempos que fueron significativamente reducidos en el tercer experimento $(6 \mathrm{~cm}$. en $16 \mathrm{~min}$., o sea 2'6 minutos por centímetro, media que en el primer experimento era de 7 minutos por centímetro aproximadamente). La incidencia que, en el desarrollo de este tipo de experimentos, tiene el factor experiencia y familiaridad con las particularidades mecánicas y tecnológicas del hacha de piedra ha sido señalada entre otros por Sillitoe (1979: 151).

Hay que destacar que el desgaste visible a nivel microscópico fue muy escaso y en cualquier caso menor del previsto a tenor de las características de la pieza. Desde luego se puede alegar justificadamente que la ausencia de desgaste carece de significación dado el breve tiempo que el hacha fue utilizada. Sin embargo, en otros experimentos con hachas de piedra pulida (diorita y anfibolita), si bien empleadas sobre madera más dura (roble), se produjo una significativa abrasión del filo al cabo de un corto espacio de tiempo (entre 5 y 21 minutos de trabajo) (Olausson, 1982-83: 42 y 44). Por otra parte, se podría esperar que la inexperiencia del que suscribe en este tipo de tareas provocara una mayor usura del filo debido a un impulso excesivo o a errores en el ángulo de incidencia del hacha sobre el tronco.

A lo largo del experimento ha quedado patente la relevancia que tiene un adecuado enmangue para la consecución de una herramienta eficiente. En el caso presente, creemos que hubiese sido necesario probablemente un mayor grosor de la zona del mango donde se insertaba el hacha con la finalidad de evitar el astillado del alvéolo. A este respecto, Harding y Young plantean la conveniencia de que el plano del alvéolo se disponga de forma ortogonal respecto a los anillos de la madera, aunque las observaciones efectuadas en Seeberg parecen indicar lo contrario (Olausson, 1982: 44). Otra posibilidad para evitar la rotura del mango es la apuntada por Jørgensen (1985: 25), haciendo que sólo los lados del hacha estén en estrecho contacto con la vaina, mientras se deja un espacio entre esta última y las caras de la pieza.

La acción del hacha configuró sobre el tronco del árbol un corte de una forma muy peculiar, con un perfil transversal en triángulo rectángulo, estando la base en la parte inferior del tajo. Esta particular disposición es muy similar a la documentada en postes recuperados en Burgäschisee-Süd (Suiza) (Müller-Beck, 1963: 130 y fig. 276). La razón para que los cortes practicados en el árbol sean

T. P., 1992, $\mathrm{n}^{2} 49$ 
tan abiertos estriba en la necesidad de evitar que el hacha quede atascada en el tajo con el consiguiente peligro de fractura de aquella.

Con anterioridad a su utilización, el hacha fue examinada bajo el microscopio, aunque con cierta dificultad debido a que el enmangue hacia incómoda su colocación en el porta. Utilizando entre 50x y 100x se pudo observar la existencia de finas estrías perpendiculares al filo prolongándose más allá de la zona activa. A todo lo ancho de la superficie de la pieza destacaban pequeñas áreas pulimentadas y el filo aparecía en buen estado, con sólo algunas microlascas visibles.

Tras la utilización, podía observarse a simple vista la presencia de residuos orgánicos (savia o pequeñas fibras leñosas) en un área que llegaba aproximadamente hasta unos $35 \mathrm{~mm}$. de distancia del filo. Por otra parte, se detectaba una zona lustrosa yendo a lo largo del corte en una banda con un mayor desarrollo hacia uno de los vértices de éste, donde alcanzaba unos $7 \mathrm{~mm}$. de anchura. Dicha pátina brillante era algo más marcada y extensa en una de las caras (Fig. 1). Hay que resaltar que la asimetría tanto en la distribución sobre cada faceta del filo como entre ambas facetas concuerda con la mecánica de utilización del hacha, pues al incidir ésta sobre la madera en un ángulo bastante agudo, una de las caras de la zona activa está en contacto más prolongado con la materia trabajada, mientras que el desplazamiento aproximadamente semicircular que sigue la herramienta en el curso de su empleo hace que el filo se interne dentro de la madera más profundamente por uno de sus extremos.

El análisis microscópico se vio complicado a causa de dos circunstancias: el tamaño de la pieza, excesivo para trabajar con holgura, y la incurvación de la superficie del hacha, lo cual hacía muy difícil una visión nítida, incluso con pocos aumentos. Se procedió previamente a limpiar las caras del hacha, sumergiendo ésta en un tanque ultrasónico durante 60 minutos y pasando a continuación un algodón, impregnado en alcohol metílico. El corte estaba en buen estado aunque algo machacado y con algunos lascados. En la zona activa, donde el lustre se apreciaba a simple vista, aparecía pulimento, a veces con una estructuración lineal perpendicular al filo y se distinguían asimismo finas estrías entrecruzándose, aunque con una orientación genéricamente oblícua respecto al corte. Dada la clara persistencia de restos orgánicos en las inmediaciones de la zona activa, procedimos a sumergir la pieza en una solución de detergente enzimático, durante sendos períodos de 1 hora cada uno, separados por observaciones microscópicas. Tras dicha limpieza solo se observaba micropulidos en las partes más altas de la superficie, una disposición que se corresponde más bien con el roce contra un material duro que con madera. En otras palabras, las alteraciones señaladas en esas zonas serían probablemente el resultado del pulimento previo de la pieza, hipótesis que se refuerza al documentar observaciones idénticas en áreas alejadas del filo, en pleno centro del hacha.

\section{CONCLUSIONES}

El carácter de primer ensayo de este experimento y lo limitado de su duración nos obligan a ser prudentes en cuanto a los resultados alcanzados, pero permiten avanzar algunas propuestas y observaciones de orden general:

Un trabajo de tala durante corto tiempo y sobre una madera de dureza media no produjo un nivel significativo de desgaste sobre el filo y zonas adyacentes, sea a nivel macroscópico o incluso microscópico, esto a pesar de habernos movido dentro de un umbral que alcanzaba los 200 aumentos.

La patina brillante, visible después de la utilización en las inmediaciones del corte, se distribuía a lo largo de éste de una forma asimétrica, acorde con la mecánica del uso de un hacha taladora, según diversos autores. Por otro lado una vez efectuada una limpieza, este lustre desapareció por completo, demostrándose de esa forma que no era consecuencia de un proceso de abrasión de la capa superficial del hacha sino que probablemente se había formado por residuos orgánicos 
amalgamados por la combinación del calor y presión resultantes del impacto o roce de la zona activa sobre la madera.

Pudimos reconocer la existencia de estrías, a veces dispuestas de forma oblicua en relación al eje de la pieza, con anterioridad a su utilización efectiva; asimismo se hallaban algunos microlascados previos. Esto hace necesario el establecimiento de criterios discriminantes, basados en experimentación con réplicas actuales para definir, si ello es factible, cuáles de esas características son debidas al uso y cuáles al proceso de manufactura. A tal fin es absolutamente imprescindible llevar a cabo observaciones a nivel macro y microscópico del utillaje experimental antes de proceder a su utilización.

\section{BIBLIOGRAFIA}

aira Rodríguez, M. J., SaA Otero, P. y Taboada Castro, T. (1989): Estudios paleobotánicos y edafológicos en yacimientos arqueológicos de Galicia, Santiago de Compostela.

Cauvin, J. (1968): «Les outillages neolithiques de Byblos et du littoral libanais». Fouilles de Byblos, 4, Paris.

Chouza Mata, M. y CID GonzÁLez, R. (1985): As árbores. Santiago de Compostela.

- DeliBes DE CASTRO, G. (1974): «Contribución al estudio de las funciones del hacha pulimentada. Resultados de la aplicación del sistema Semenov a 130 ejemplares de Tierra de Camposn. Zephyrus, 25: 151-154.

DETEV, P. (1960): «Essai d'etablir une distinction entre las haches et les ciseaux, les herminettes, les houes et les socs de l'epoque neolithiquew. Annuaire du Musée National Archéologique du Plovdiv, 4: 61-74.

Fábregas VALCARCE, R. (1991): Estudio de los ajuares líticos de las sepulturas megalíticas de Galicia y Norte de Portugal. Tesis Doctoral microfilmada. U.N.E.D., Madrid.

HARDING, F. (1983): «An experiment to produce a ground flint axe». En G. Sieveking y M. H. Newcomer (eds.). The Human Uses of Flint and Chert. Papers presented at the 4th International Flint Symposium, Brighton: 37-42.

HARDING, A. y YouNG, R. (1979): «Reconstruction of the hafting methods and function of stone implementa». T. H. McK. Clough y W. A. Cummins (eds.), Stone Axe Studies, C.B.A. Research Report, 23: 102-105.

IVERSEN, J. (1956): «Forest clearance in the Stone Age». Scientific American, 194-3: 36-41.

JøRGENSEN, S. (1985): Tree-Felling in Draved. Copenhague.

KANTMAN, S. (1969-70): «A microanalytic study of some ground stone artefacts from Tilmen Höyük and GedïkliKarahöyưk (Southeastern Anatolia). Contributions to functional typologyn. Anatolica, 3: 139-145.

MADSEN, B. (1984): «Flint axe manufacture in the Neolithic: Experiments with Grinding and Polishing of ThinButted Flint Axes". Journal of Danish Archaeology, 3: 47-62.

Muller-Beck, H. (1965): Seeberg Burgäschisee-Süd. Holzgeräte ond Holzbearbeitung. Acta Bernensia, 2-5.

NILSON, S. (1868): The primitive inhabitants of Scandinavia. Londres.

OLAUSSON, D. S. (1982): «Lithic technological analysis of the thin-butted flint axe». Acta Archaeologica, 53: 1-87.

- (1982-1983): «Flint and groundstone axes in the Scanian Neolithic. An evaluation of raw materials based on experimentw. Scripta Minora, 2: 7-66.

QUENTE, P. (1914): «Steinzeitliche Ackerbaugeräte aus der Ostprignitz, Erdhacken und Pflüge, und ihre Schaftungsmöglichkeitn. Praehistorische Zeitschrift, 6: 180-187.

ROODENBERG, J. J. (1982): «Traces d'utilisation sur les haches polies de Bouqras (Syrie)». M. C. Cauvin (ed.), Traces d'utilisation sur les outils néolithiques du Proche Orient. Travaux de la Maison de l'Orient, 5: 177-188.

SEHESTED, N. F. B. (1884): Praktiske forsøg, Archaeologiske Unders Øgelser 1878-1881. Copenhague.

Semenov, S. A. (1981): Tecnología Prehistórica. Madrid.

SILLITOE, P. (1979): «Stone versus steel». Mankind, 12: 151-161.

SONNENFELD, J. (1962): «Interpreting the function of primitive implements». American Antiquity, 28-1: 56-65.

StelCL, J. y Malina, J. (1970): "Anwendung der Petrographie in der Archäeologie». Folia Facultatis Scientiarum Naturalium Universitatis Purkynianae Brunensis, Geologia, 11-5. 

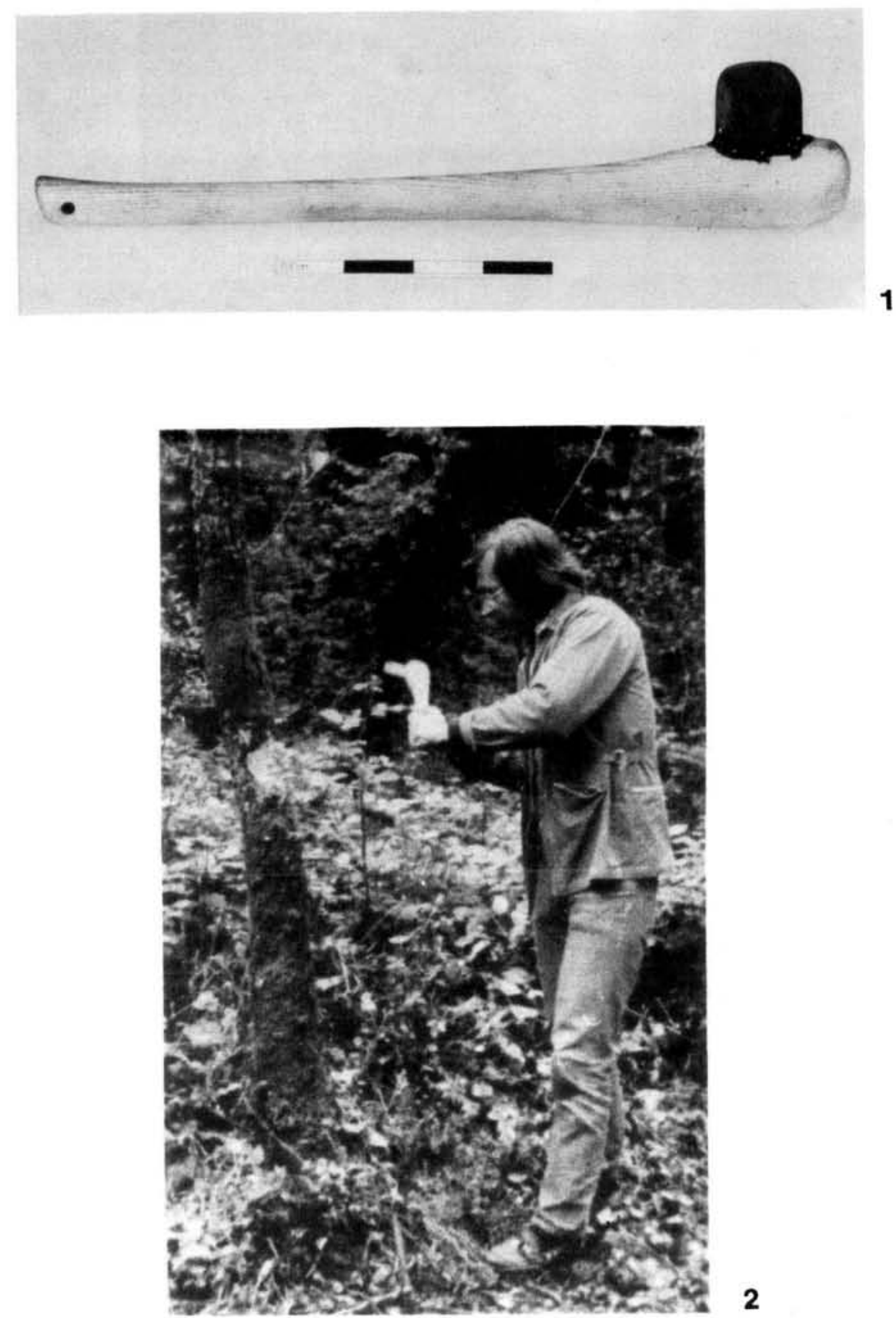

2

1.-Hacha enmangada, antes de su utilización. 2. El Dr. Newcomer mostrando la técnica utilizada para cortar un árbol con un hacha de piedra pulida. 


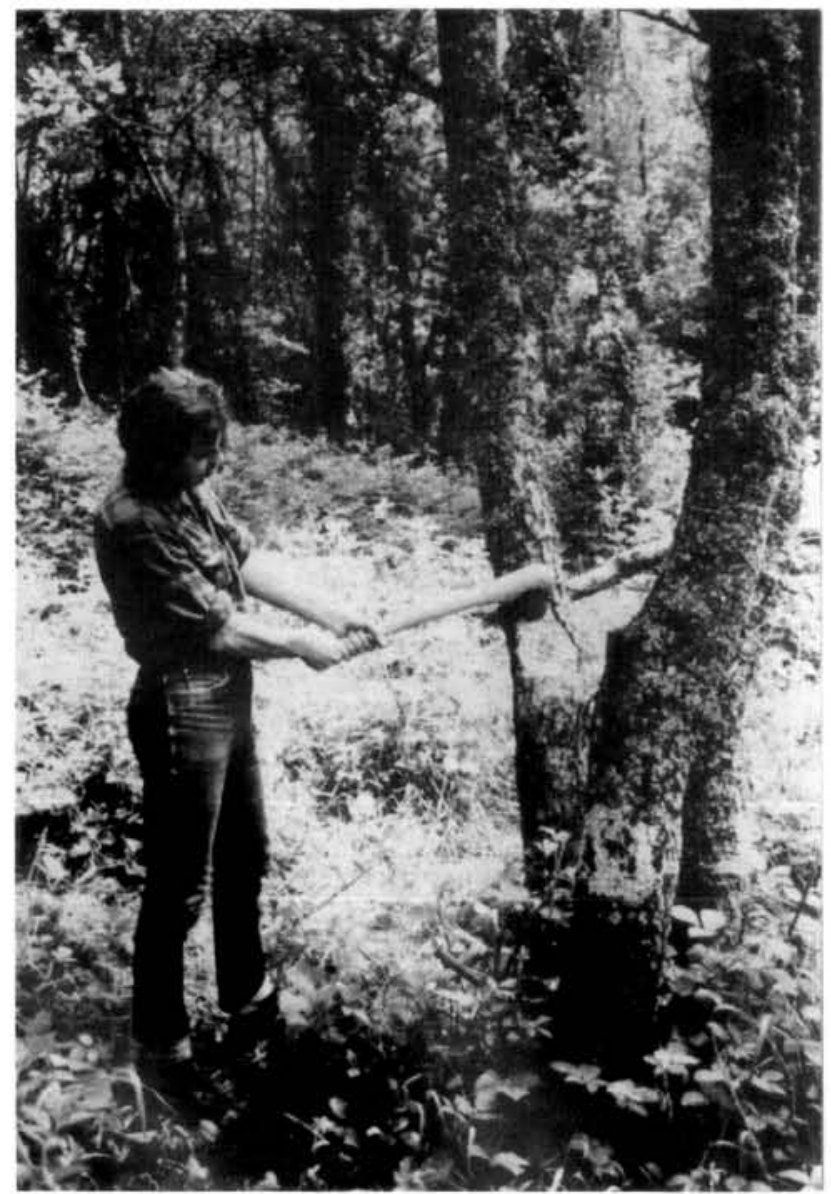

1

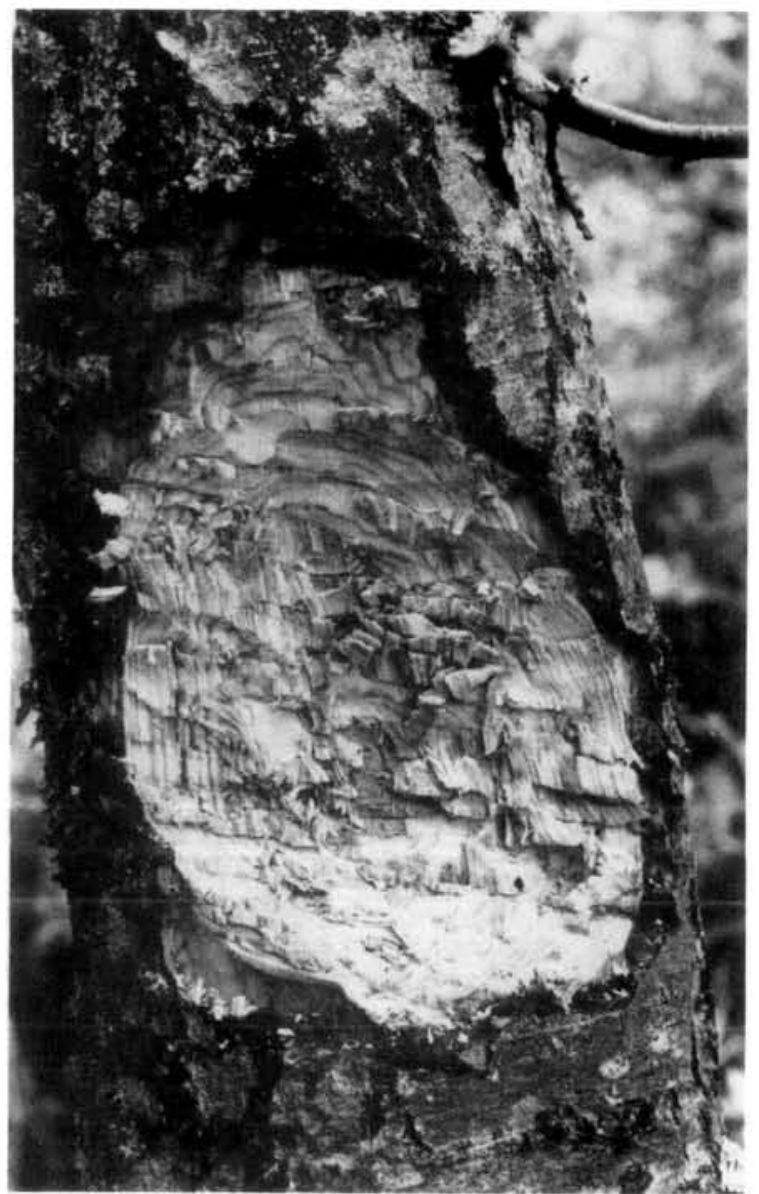

2

1.-El Dr. Grace indicando el ángulo aproximado en que el hacha golpea el tronco.- 2. Aspecto del corte efectuado en el curso del segundo experimento.

T. P., 1992, $\mathrm{n}^{\circ} 49$ 

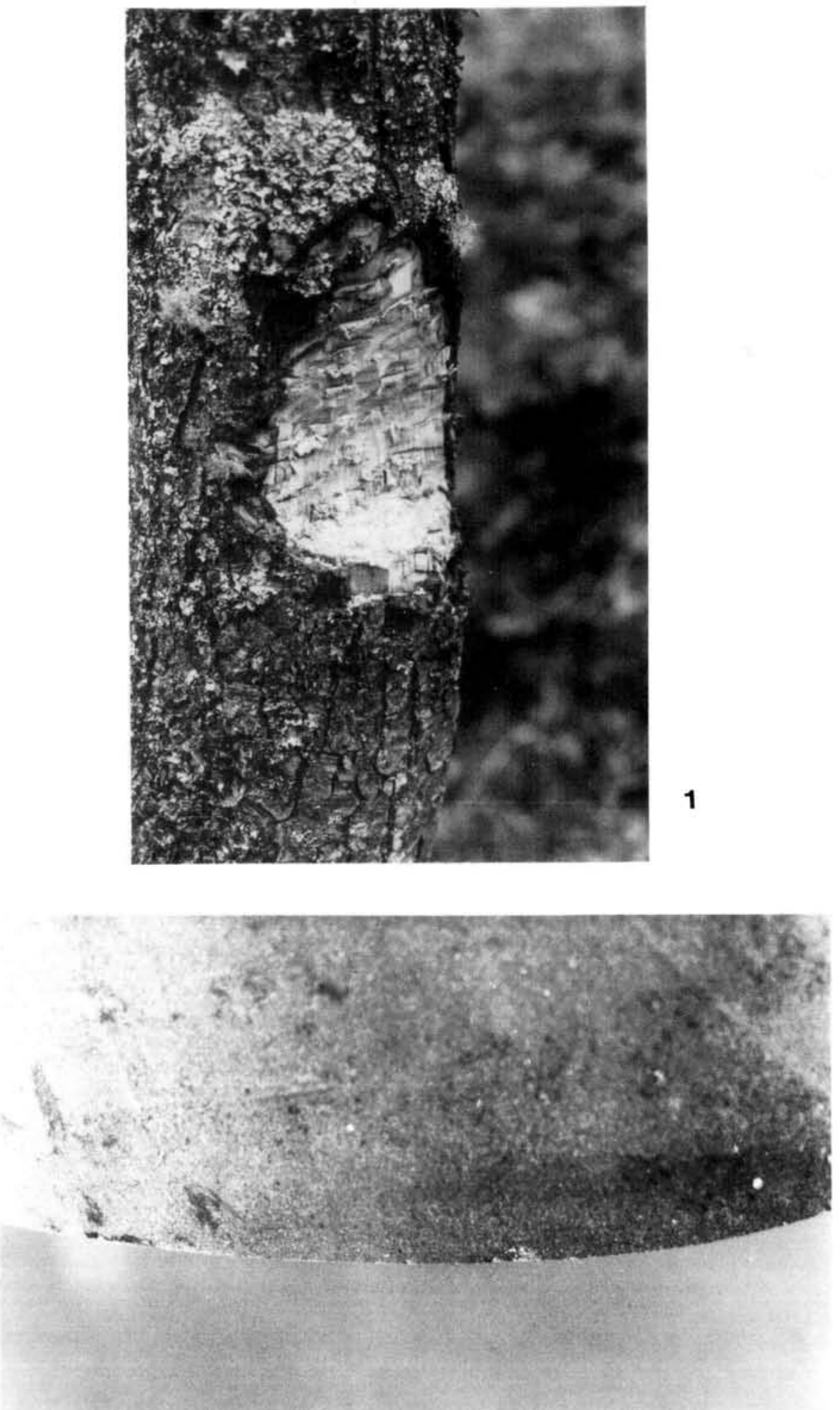

2

1.-Aspecto del corte tras la interrupción del tercer experimento. 2.-Zona activa del hacha después del experimento.

T. P., 1992, no 49 